\title{
A Novel Leakage Reduction Technique for Ultra-low Power in VLSI Circuit
}

\author{
Md. Tauseef \\ ECE, Dept.LNCT, Bhopal, \\ India
}

\author{
Sudeep Sharma \\ ECE, Dept.LNCT, Bhopal, \\ India
}

\author{
Rita Jain \\ ECE, Dept.LNCT, Bhopal, \\ India
}

\begin{abstract}
The modern portable devices demands ultra-low power consumption due to the limited battery size. Major concerns of VLSI designers were high performance with minimal size earlier. The fast growth in portable computing and wireless communication has led to the power dissipation along with heating. The leakage causes static power consumption is exceeding the dynamic power in the sub-nanometer designs. In order to maintain the performance of the chip along with high driving capability at lower supply voltage, the $\mathrm{V}_{\mathrm{TH}}$ is reduced. However, the Threshold Voltage $\left(\mathrm{V}_{\mathrm{TH}}\right)$ scaling results increase of the Subthreshold Leakage Current $\left(\mathrm{I}_{\mathrm{SUB}}\right)$ as $\mathrm{V}_{\mathrm{TH}}$ is exponentially proportional to $\mathrm{I}_{\mathrm{SUB}}$. Power consumption has become primary design issue and needs suitable power management in the design of digital circuits where switching and standby mode affects the performance of system. In this paper we have calculate the leakage power consumption of conventional gates and proposed leakage reduction techniques over various gates at $45 \mathrm{~nm}$ and $32 \mathrm{~nm}$ process technology with supply voltage of $0.9 \mathrm{v}$ and $0.8 \mathrm{~V}$ by using HSPICE simulator at $100 \mathrm{MHz}$ frequency.
\end{abstract}

\section{Keywords}

Low Power Design, Leakage reduction, Integrated Circuits, VLSI.

\section{INTRODUCTION}

In a digital CMOS circuits dynamic power dissipation is major concern in total power dissipation. The most effective approach for dynamic power reduction is reducing the supply voltage. However circuit performance degraded as reducing the supply voltage alone. To improve the circuit performance threshold voltage of transistor should be decreases with decrease in supply voltage. Lower size transistor leaks more power through the source during idle state of the device which directly affects the battery life. Now customer demands portable devices which give performance same as nonportable devices which not just occupy less area, should also have more battery life. In this way designer switch to low power CMOS VLSI design where the supply voltage of the IC is reduced which in turn reduces the switching power dissipation in micron devices which was the main cause of total power dissipation [1]. Subthreshold leakage power dissipation is becoming more dominating among all leakage sources of the device below $90 \mathrm{~nm}$ technology. The leakage power can give approximately $42 \%$ of the overall power dissipation in CMOS VLSI circuit design in 90nm technology [18]. In this research work the cause of leakage in CMOS circuits is explored and some gates of standard cell library are modified which reduce the leakage during standby mode without technology modification. [1]. Subthreshold leakage power dissipation is becoming more dominating among all leakage sources of the device below $90 \mathrm{~nm}$ technology. In this research work the cause of leakage in CMOS circuits is explored and some gates of standard cell library are modified which reduce the leakage during standby mode without technology modification. [3].In this paper the leakage current is reducing in pull down network on the Circuit. For the suggested technique uses Two PMOS in Pull Down network.

As with the scaling down of the technology, it results in the increment of leakage current in transistor that includes gate drain leakage, gate-oxide tunneling, drain-induced barrier lowering and weak inversion effect. It is observed that the low threshold voltages, gate leakage and sub-threshold voltages are the dominant sources of leakage current in deep submicron meter devices. Effect of such sources will increase with technology scaling. The GIDL and BTBT (base to base tunneling) may also have a significant effect on advanced CMOS devices. The solution should be considered both at circuit level and process technology level in deep sub-micron meter CMOS circuits. It is a run time leakage reduction technique which utilizes the body (substrate) terminal of the MOS transistor to dynamically modify the $\mathrm{V}_{\mathrm{TH}}$ of a transistor during circuit operationThe positive charge on the gate is balanced by the sum of the electronic charge in the inversion layer and the negative ionic charge in the depletion region. During RBB of a MOSFET, the width of the depletion region beneath the gate increases difference between the source and body terminals $\left(\mathrm{V}_{\mathrm{SB}}\right)[4,5]$.

The objective is to minimize the leakage power consumption with technology scaling with large number of gates per chip is to develop a run time leakage reduction technique which focuses stacking effect of transistor and circuit using high $-\mathrm{K}$ (high dielectric) library to minimize $\mathrm{I}_{\mathrm{GATE}}$.

Independent of technology variation, and taking advantages of stacking effect.

- Needs no of any extra supply voltage and additional controller like RBB technique.

- Keeps trade-off between area, power and delay.

- Solves in polynomial time (less Time complexity).

\section{RELATED WORK}

There are several techniques to control leakage current at transistor level design circuits. Body biasing is one of the techniques to reduce leakage current of circuit. INDEP approach [1] is the technique which mitigates the leakage current in nanoscale circuit. This technique has two extra inserted transistors between pull up and pull down networks which are input logic dependent. This technique is used for static CMOS circuit and has sufficient delay penalty.

These guidelines are presented in general terms so that they can be adopted for any application and process technology.

The power consumption of a logic gate is given by:- 


$$
\mathrm{P}_{\text {avg } / \text { gate }}=\mathrm{P}_{\text {switching }}+\mathrm{P}_{\text {short circuit }}+\mathrm{P}_{\text {leakage }}
$$

Where $\mathrm{P}_{\text {switching }}$ is the power consumed due to charging and discharging of the circuit capacitances, $\mathrm{P}_{\text {short }}$ circuit is the power consumed due to the short circuit between $\mathrm{V}_{\mathrm{DD}}$ and ground during output transitions and $\mathrm{P}$ leakage is a leakage power consumption. Static (leakage) power $\left(P_{\text {leakage }}\right)$ is consumed by the circuit leakage current in its steady state i.e., when the circuit is "powered-on". Collectively, dynamic power and short circuit power are called switching power. Switching power dissipates when logic state of input signal changes in CMOS circuits.

Subthreshold leakage current $\left(\mathrm{I}_{\mathrm{sub}}\right.$ The sub threshold current flows due to three main reasons: Drain Induced Barrier Lowering (DIBL) effect, weak inversion effect and the direct punch-through of the electrons between drain and source. The DIBL effect occurs at higher drain voltages where threshold voltage of transistor reduces. Depletion region of the $\mathrm{p}-\mathrm{n}$ junction between the drain and body increases with the increase in drain voltages which increases more under the gate voltage.

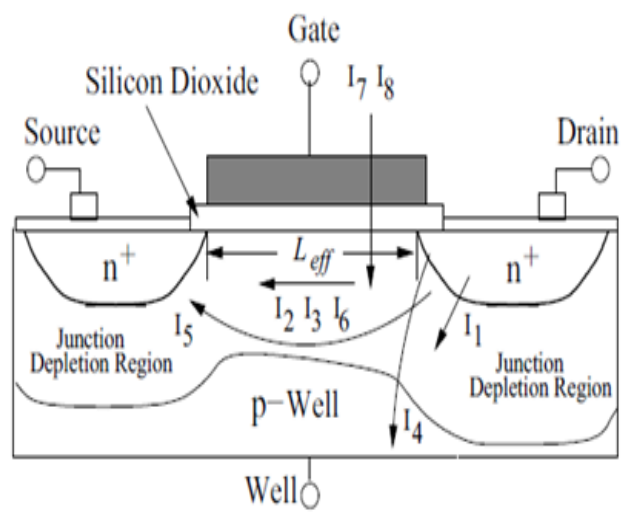

Fig.1: Leakage Mechanism in Short-Channel NMOS Transistor

The source injects carriers into the channel surface (independent of gate voltage). Narrow width of the transistor can also modulate the threshold voltage and the subthreshold current.

$$
I_{s u b}=\mu_{0} \cdot C_{o x} \cdot \frac{W}{L} \cdot V^{2} \cdot e^{1.8} \cdot e^{\frac{\left(V_{g s}-V_{T}\right)}{n V}}
$$

where, $\mu_{0}$ is the zero bias mobility, oxide capacitance is represents as $\mathrm{C}_{\mathrm{ox}}$, and (W/L) represents the width to the length ratio of the leaking MOS device. The sub threshold current flows due to three main reasons: Drain Induced Barrier Lowering (DIBL) effect, weak inversion effect and the direct punch-through of the electrons between drain and source. The DIBL effect occurs at higher drain voltages where threshold voltage of transistor reduces. Depletion region of the p-n junction between the drain and body increases with the increase in drain voltages which increases more under the gate voltage. Responsibility to balance the electron charges in depletion region is more on drain voltage rather than gate voltage.

\section{Gate oxide tunnelling current}

Tunnelling through gate oxide occurs because thickness of gate oxide layer is gradually reduced as technology is reducing [7 The GIDL and BTBT (base to base tunneling) may also have a significant effect on advanced CMOS devices. The solution should be considered both at circuit level and process technology level in deep sub-micron meter CMOS circuits. At circuit level, variable threshold, dynamic threshold, dual threshold, multi-threshold and transistor stacking techniques can effectively reduce the leakage current in memory and high performance CMOS circuits. At the process technology level, halo doping and retrograde techniques are used to reduce leakage current. Such well engineering techniques also improve short-channel characteristics.

\section{Band to band tunnelling current}

While studying about reverse leakage current we came to know that within the geometry of the device, some junction diodes are present. Because of the reverse biased diode a voltage is developed across the diode and high electric field across this reverse biased $p-n$ junction cause significant current known as Band to band tunnelling current. This band to band tunnelling current are larger than reverse biased leakage current in deep submicron technology.

\section{Gate induced drain leakage current}

Gate induced drain leakage current occurs due to high electric field in the drain junction. This current occurs because of short channel length due to which a high electric field is created. Even a small voltage creates a large electric field.

\section{Gate current due to hot carrier injection}

Another kind of gate leakage is known as the gate current due to hot carrier injection. For a very small gate voltage because the smaller thickness of the Silicon dioxide layer electric field becomes so high that it creates electrons of very high energy known as hot electrons (hot carriers). Those hot electrons acquire a very high energy that they can pass through the Silicon dioxide layer

\subsection{Dual Threshold (Dual- $\left.\mathbf{V}_{\mathrm{TH}}\right)$ Technique}

Dual-Threshold CMOS technique is frequently used at subsystem design level. For this technique a sub-system is implemented with low $\mathrm{V}_{\mathrm{TH}}$ transistors or a high $\mathrm{V}_{\mathrm{TH}}$ transistors depending upon whether they lie in the critical path or not [7-8]. Here, various algorithms are used to take decision regarding critical path of the circuit. If a sub-system lies on the critical path, low $\mathrm{V}_{\mathrm{TH}}$ transistors are used for implementing the sub-system design while High $\mathrm{V}_{\mathrm{TH}}$ transistors based sub-systems are used on non-critical paths. For this kind of arrangement they would not affect the circuit timing and hence performance as well as leakage optimization can be achieved. Two kinds of algorithm are used such as exact or Heuristics. 


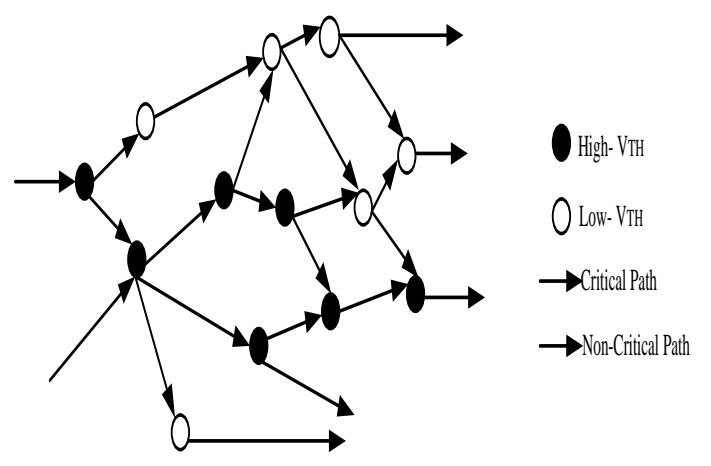

Fig. 2. Dual- $\mathrm{V}_{\mathrm{TH}}$ Techniques

\subsection{Transistor Stacking Technique}

Transistor stacking is a runtime leakage reduction technique in which a single transistor divide into two half size transistor. The purpose of this kind of arrangement is to increase the number of off transistor in stack. If two transistors are off instead of single off transistor highly reduces the leakage. I $_{\mathrm{SUB}}$ is exponentially depends on the potential at each terminal every in CMOS. Fig.3. depicts the effect of self-reverse bias when gate terminal potential is at ground, and variation of drain current is occur [9].
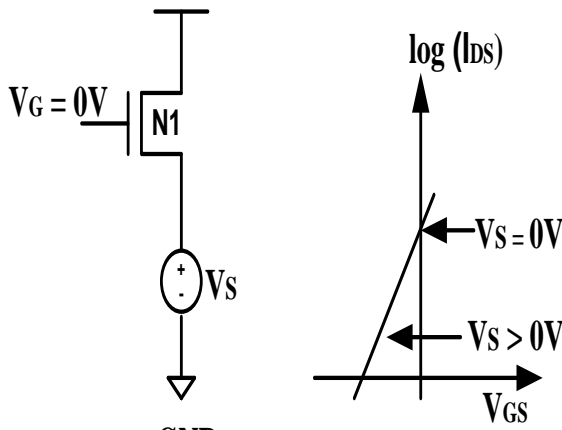

GND

Fig.3.Self-Reverse biasing effects on $I_{S U B}$

When a source terminal is biased of an NMOS transistor, it reduces $\mathrm{I}_{\mathrm{SUB}}$ exponentially due to the following facts: Fig.4 stacking of two NMOS transistor. Here width of these NMOS are $\mathrm{W} / 2, \mathrm{~W}$ is the width of original NMOS transistor.

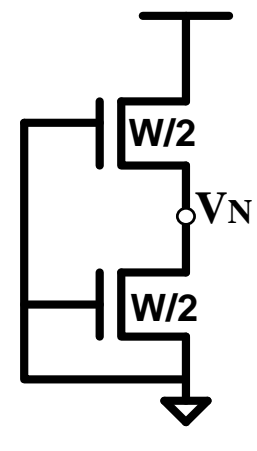

GND

Fig.4. Two NMOS Transistor Stack OFF condition

\subsection{Power Gating Technique}

Power gating technique cut-off the logic circuit from $\mathrm{v}$ and ground $\mathrm{V}_{\mathrm{dd}}$ to GND for reduction in subthreshold leakage current, which flows from the power supply towards ground due to non-ideal characteristics (finite resistance) of CMOS transistor. This technique uses the power supply voltage as the primary source for minimizing leakage current. It inserts an extra MOS switch as a sleep transistor to cut off, or gate, a circuit from the power rails ( $\mathrm{V}_{\mathrm{DD}}$ and/or GND) during standby mode. The additional sleep switch is connected typically as header between the circuit and the $\mathrm{V}_{\mathrm{DD}}$ or as footer between the circuit and the GND [10].

\subsection{Body Bias Technique}

It is a run time leakage reduction technique which utilizes the body (substrate) terminal of the MOS transistor to dynamically modify the $\mathrm{V}_{\mathrm{TH}}$ of a transistor during circuit operation. Depending upon the polarity of the voltage difference between the source and body terminals $\left(\mathrm{V}_{\mathrm{SB}}\right)$, the $\mathrm{V}_{\mathrm{TH}}$ can be either increased. The $\mathrm{V}_{\mathrm{TH}}$ is increased when the source-to-substrate $\mathrm{p}-\mathrm{n}$ junction of a MOSFET is reversing biased called Reverse Body Biasing (RBB) [11-12]. The $\mathrm{V}_{\mathrm{TH}}$ of a MOSFET can be reduced by forward biasing the sourceto-substrate $\mathrm{p}-\mathrm{n}$ junction called Forward Body Biasing (FBB). RBB for CMOS; 45nm PTM [11] file is used here. For leakage reduction $\mathrm{RBB}$ is preferred because it increases the $\mathrm{V}_{\mathrm{TH}}$ which results leakage reduction of the logic circuit.

- Reduced complexity of logic and hence, lower capacitance, and faster speed.

- The power consumption is usually higher than conventional CMOS design, because static current always flows through logic gate whenever the pulldown network is on.

Better suited for large fan-in gates because each input connects to a single transistor, presenting a smaller load to the preceding gate.

\section{PROPOSED WORK}

In this paper, structure and operation of the proposed lowleakage-power design stack with pass transistor logic. The proposed circuit is compared with well-known previous approaches, i.e., Conventional Gates. In over proposed circuit we have introduce two technique stack approach with pass transistor approach to reduce the leakage power consumption in the circuit. Here we use two NMOS pass transistor is places below pull up network and PMOS transistor place parallel to the NMOS transistor in between pull up network and pull down network. The Pull up transistor Turn ON NMOS pass transistor and Pull down transistor turns ON PMOS pass transistor during active mode of the circuit, during sleep both the pass transistor turns of and rail the network from the supply voltage which help in reduction of the leakage power. Similar action also repeats in pull down network the while interchanging the pass transistor NMOS transistor provide the stacking effect (Fig.5.). To maintain the value "0" in sleep mode operation and PMOS pass transistor connect parallel with NMOS transistor. To maintain an output value to "0" PMOS transistor connected to GND in sleep mode. To achieve proper Logic at the output NMOS transistor is connect to Vdd and PMOS transistor is connect to GND. The stacking of the transistor reduces the leakage power in proposed approach. To maintain the proper high logic insert NMOS transistor parallel to PMOS stacked transistor in pull up network, to connect sleep transistor to Vdd to the pull up 
network. In sleep mode, this NMOS transistor connects Vdd to the pull up network when sleep transistor cut off.

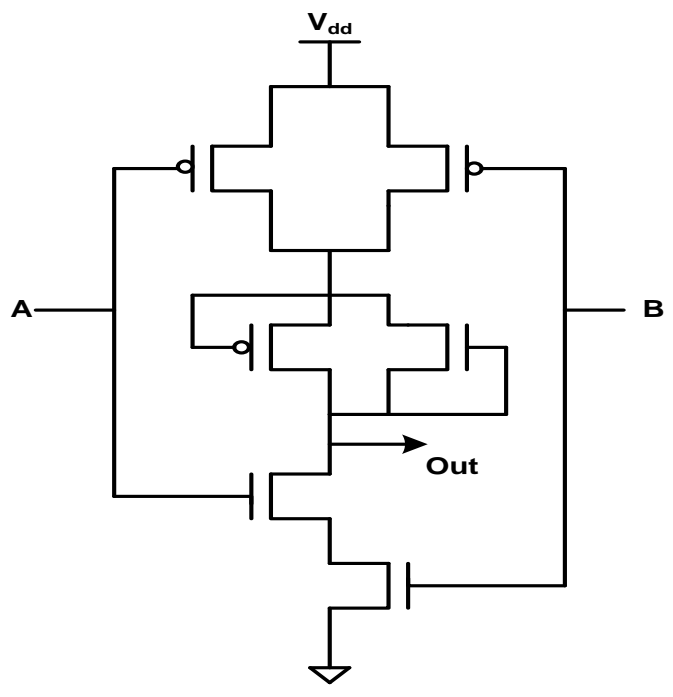

Fig.5. Proposed Circuit

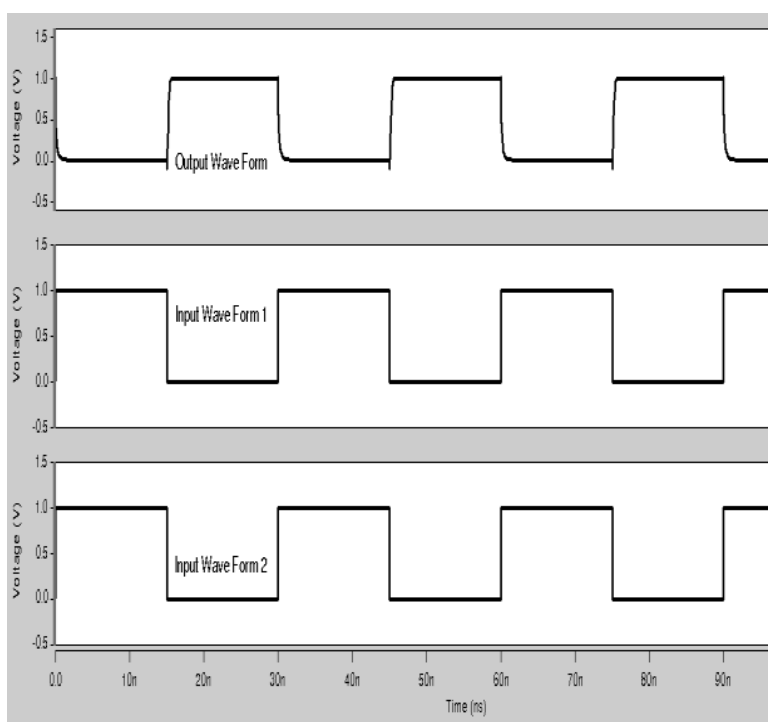

Fig.6. Output Wave form of Proposed Circuit

\section{RESULTS AND DISCUSSION}

Leakage current for Proposed circuit is calculated by using Berkley Predictive Technology Module (BPTM) in HSPICE simulator using $45 \mathrm{~nm}$ and $32 \mathrm{~nm}$ process technology with supply voltage of $0.9 \mathrm{v}$ and $0.8 \mathrm{~V}$ at $10 \mathrm{MHz}$ frequency and $\mathrm{C}_{\mathrm{L}}=1 \mathrm{pf}$. Leakage power of conventional gate is compared with Proposed technique implemented in with all the input vector combination at $25^{\circ} \mathrm{C}$ and $100^{\circ} \mathrm{C}$ temperature respectively. Transient analysis of proposed technique with Nand gate. It is observe that output wave form provides proper logic.

Table I. Dynamic Power at $45 \mathrm{~nm} 25^{\circ} \mathrm{C}$

\begin{tabular}{|l|c|c|c|c|c|}
\hline Gates & Average & \multicolumn{3}{|c|}{ Delay $(\mathrm{pS})$} & \multirow{2}{*}{ PDP } \\
\cline { 3 - 5 } & Power $(\mu \mathrm{W})$ & $\mathrm{T}_{\mathrm{R}}$ & $\mathrm{T}_{\mathrm{F}}$ & $\mathrm{T}_{\mathrm{R}}+\mathrm{T}_{\mathrm{F}}=\mathrm{T}_{\text {Total }}$ & \\
\hline NOT & 0.2057 & 4.847 & 5.181 & 5.014 & 1.031 \\
\hline AND & 0.4163 & 10.96 & 5.424 & 8.192 & 3.410 \\
\hline NAND & 0.2627 & 8.455 & 3.352 & 5.903 & 1.550 \\
\hline NOR & 0.2336 & 9.279 & 3.094 & 6.186 & 1.445 \\
\hline EXOR & 0.3595 & 6.247 & 6.572 & 6.409 & 2.304 \\
\hline
\end{tabular}

Table II. Dynamic Power at $45 \mathrm{~nm} 100^{\circ} \mathrm{C}$

\begin{tabular}{|l|l|l|l|l|l|}
\hline Gates & Average & \multicolumn{5}{|l|}{ Delay } & PDP \\
\cline { 3 - 5 } & Power & $\mathrm{T}_{\mathrm{R}}$ & $\mathrm{T}_{\mathrm{F}}$ & $\mathrm{T}_{\mathrm{R}}+\mathrm{T}_{\mathrm{F}}=\mathrm{T}_{\text {Total }}$ & \\
\hline NOT & 0.3048 & 4.671 & 5.418 & 5.044 & 1.537 \\
\hline AND & 0.6534 & 11.21 & 5.318 & 8.264 & 5.399 \\
\hline NAND & 0.3901 & 8.374 & 2.779 & 5.576 & 2.175 \\
\hline NOR & 0.3205 & 9.810 & 3.173 & 6.491 & 2.080 \\
\hline EXOR & 0.4065 & 6.046 & 6.874 & 6.362 & 2.586 \\
\hline
\end{tabular}

Table III. Dynamic Power at $32 \mathrm{~nm} 25^{\circ} \mathrm{C}$

\begin{tabular}{|l|l|l|l|l|l|}
\hline Gates & Average & \multicolumn{3}{|l|}{ Delay } & \multirow{2}{*}{ PDP } \\
\cline { 3 - 5 } & Power & $\mathrm{T}_{\mathrm{R}}$ & $\mathrm{T}_{\mathrm{F}}$ & $\mathrm{T}_{\mathrm{R}}+\mathrm{T}_{\mathrm{F}}=\mathrm{T}_{\text {Total }}$ & \\
\hline NOT & 0.1440 & 5.382 & 5.612 & 5.497 & 0.791 \\
\hline AND & 0.2708 & 11.19 & 5.658 & 8.424 & 2.281 \\
\hline NAND & 0.1862 & 9.422 & 2.694 & 6.058 & 1.127 \\
\hline NOR & 0.1661 & 10.73 & 3.571 & 7.150 & 1.187 \\
\hline EXOR & 0.1875 & 11.96 & 12.83 & 12.39 & 2.323 \\
\hline
\end{tabular}

Table IV. Dynamic Power at 32nm $\mathbf{1 0 0}^{\circ} \mathrm{C}$

\begin{tabular}{|c|c|c|c|c|c|}
\hline \multirow[t]{2}{*}{ Gates } & \multirow{2}{*}{$\begin{array}{l}\text { Average } \\
\text { Power }\end{array}$} & \multicolumn{3}{|c|}{ Delay } & \multirow[t]{2}{*}{ PDP } \\
\hline & & $T_{R}$ & $\mathrm{~T}_{\mathrm{F}}$ & $\mathrm{T}_{\mathrm{R}}+\mathrm{T}_{\mathrm{F}}=\mathrm{T}_{\text {Total }}$ & \\
\hline NOT & 0.2210 & 5.117 & 5.810 & 5.463 & 1.207 \\
\hline AND & 0.4534 & 11.15 & 5.940 & 8.545 & 3.874 \\
\hline NAND & 0.2860 & 9.604 & 3.053 & 6.328 & 1.809 \\
\hline NOR & 0.2328 & 11.06 & 3.235 & 7.148 & 1.664 \\
\hline EXOR & 0.2865 & 10.93 & 11.58 & 11.25 & 3.223 \\
\hline
\end{tabular}

Table V. Leakage Power Consumption at $45 \mathrm{~nm}$ at $25^{\circ} \mathrm{C}$

\begin{tabular}{|l|c|c|c|c|}
\hline Gates & \multicolumn{5}{|c|}{ Leakage Power Consumption at $45 \mathrm{~nm}$} \\
\hline & 00 & 01 & 10 & 11 \\
\hline NOT & 21.83 & 75.23 & & \\
\hline AND & 60.45 & 109.1 & 76.07 & 172.8 \\
\hline NAND & 31.46 & 149.8 & 103.6 & 150.4 \\
\hline NOR & 43.63 & 83.11 & 72.86 & 111.8 \\
\hline EXOR & 155.9 & 108.4 & 108.4 & 155.9 \\
\hline
\end{tabular}

Table VI. Leakage Power Consumption at $45 \mathrm{~nm}$ at $100^{\circ} \mathrm{C}$

\begin{tabular}{|l|c|c|c|c|}
\hline Gates & \multicolumn{4}{|c|}{ Leakage Power Consumption at 45nm } \\
\hline & 00 & 01 & 10 & 11 \\
\hline NOT & 52.00 & 83.32 & & \\
\hline AND & 119.3 & 188.6 & 152.2 & 218.9 \\
\hline NAND & 39.88 & 260.2 & 154.34 & 166.5 \\
\hline NOR & 103.8 & 95.12 & 80.98 & 114.8 \\
\hline EXOR & 176.0 & 166.5 & 166.5 & 176.0 \\
\hline
\end{tabular}

Table VII. Leakage Power Consumption at $32 \mathrm{~nm}$ at $25^{\circ} \mathrm{C}$

\begin{tabular}{|l|l|l|l|l|}
\hline Gates & \multicolumn{4}{|l|}{ Leakage Power Consumption at 32nm } \\
\hline & 00 & 01 & 10 & 11 \\
\hline NOT & 10.14 & 24.88 & & \\
\hline AND & 21.34 & 48.66 & 30.00 & 59.92 \\
\hline NAND & 3.921 & 31.21 & 12.57 & 49.73 \\
\hline NOR & 20.26 & 24.97 & 24.33 & 42.90 \\
\hline EXOR & 49.28 & 43.70 & 43.70 & 49.28 \\
\hline
\end{tabular}

Table VIII. Leakage Power Consumption at $32 \mathrm{~nm}$ at $100^{\circ}$ C

\begin{tabular}{|l|l|l|l|l|}
\hline Gates & \multicolumn{4}{|l|}{ Leakage Power Consumption (nW) } \\
\hline & 00 & 01 & 10 & 11 \\
\hline NOT & 73.87 & 44.98 & & \\
\hline AND & 116.1 & 185.1 & 163.9 & 164.4 \\
\hline
\end{tabular}




\begin{tabular}{|l|l|l|l|l|}
\hline NAND & 28.07 & 96.31 & 75.11 & 89.80 \\
\hline NOR & 146.8 & 44.88 & 42.66 & 51.24 \\
\hline EXOR & 87.44 & 170.98 & 170.98 & 87.44 \\
\hline
\end{tabular}

Table IX. Proposed Dynamic Power at $45 \mathrm{~nm} 25^{\circ} \mathrm{C}$

\begin{tabular}{|l|l|l|l|l|l|}
\hline Gates & Average & \multicolumn{3}{|l|}{ Delay $(\mathrm{pS})$} & PDP \\
\cline { 3 - 5 } & Power $(\mu \mathrm{W})$ & $\mathrm{T}_{\mathrm{R}}$ & $\mathrm{T}_{\mathrm{F}}$ & $\mathrm{T}_{\mathrm{R}}+\mathrm{T}_{\mathrm{F}}=\mathrm{T}_{\text {Total }}$ & \\
\hline NOT & 0.1727 & 1.912 & 6.525 & 4.218 & 0.728 \\
\hline AND & 0.4039 & 6.271 & 5.838 & 6.054 & 2.445 \\
\hline NAND & 0.2373 & 7.560 & 6.466 & 7.013 & 1.664 \\
\hline NOR & 0.2143 & 1.183 & 11.76 & 6.471 & 1.386 \\
\hline EXOR & 0.2831 & 2.953 & 14.09 & 8.521 & 2.412 \\
\hline
\end{tabular}

Table X. Proposed Dynamic Power at $45 \mathrm{~nm} 100^{\circ} \mathrm{C}$

\begin{tabular}{|l|l|l|l|l|l|}
\hline \multirow{2}{*}{ Gates } & Average & \multicolumn{3}{|l|}{ Delay } & \multirow{2}{*}{ PDP } \\
\cline { 3 - 5 } & Power & $\mathrm{T}_{\mathrm{R}}$ & $\mathrm{T}_{\mathrm{F}}$ & $\mathrm{T}_{\mathrm{R}}+\mathrm{T}_{\mathrm{F}}=\mathrm{T}_{\text {Total }}$ & \\
\hline NOT & 0.2628 & 1.794 & 6.555 & 4.175 & 1.097 \\
\hline AND & 0.6191 & 6.614 & 5.614 & 6.114 & 3.785 \\
\hline NAND & 0.3566 & 7.167 & 6.541 & 6.854 & 2.444 \\
\hline NOR & 0.3010 & 0.958 & 12.01 & 6.484 & 1.951 \\
\hline EXOR & 0.3464 & 2.255 & 15.03 & 8.642 & 2.993 \\
\hline
\end{tabular}

Table XI. Proposed Dynamic Power at 32nm $25^{\circ} \mathrm{C}$

\begin{tabular}{|c|c|c|c|c|c|}
\hline \multirow[t]{2}{*}{ Gates } & \multirow{2}{*}{$\begin{array}{l}\text { Average } \\
\text { Power }\end{array}$} & \multicolumn{3}{|l|}{ Delay } & \multirow{2}{*}{ PDP } \\
\hline & & $\mathrm{T}_{\mathrm{R}}$ & $\mathrm{T}_{\mathrm{F}}$ & $\mathrm{T}_{\mathrm{R}}+\mathrm{T}_{\mathrm{F}}=\mathrm{T}_{\text {Total }}$ & \\
\hline NOT & 0.1255 & 3.701 & 14.00 & 8.850 & 0.464 \\
\hline AND & 0.2612 & 5.762 & 5.215 & 5.488 & 1.433 \\
\hline NAND & 0.1611 & 3.901 & 5.440 & 4.670 & 0.752 \\
\hline NOR & 0.1286 & 1.144 & 19.28 & 10.21 & 1.313 \\
\hline EXOR & 0.1345 & 7.322 & 22.91 & 15.11 & 2.032 \\
\hline
\end{tabular}

Table XII. Proposed Dynamic Power at 32nm $100^{\circ} \mathrm{C}$

\begin{tabular}{|l|l|l|l|l|l|}
\hline Gates & Average & \multicolumn{3}{|l|}{ Delay } & PDP \\
\cline { 3 - 5 } & Power & $\mathrm{T}_{\mathrm{R}}$ & $\mathrm{T}_{\mathrm{F}}$ & $\mathrm{T}_{\mathrm{R}}+\mathrm{T}_{\mathrm{F}}=\mathrm{T}_{\text {Total }}$ & \\
\hline NOT & 0.1403 & 3.555 & 14.20 & 8.877 & 1.245 \\
\hline AND & 0.4235 & 5.711 & 5.732 & 5.721 & 2.422 \\
\hline NAND & 0.2460 & 3.988 & 5.343 & 4.665 & 1.147 \\
\hline NOR & 0.1839 & 0.869 & 18.88 & 9.874 & 1.815 \\
\hline EXOR & 0.1956 & 9.961 & 29.61 & 19.78 & 3.868 \\
\hline
\end{tabular}

Table XIII. Proposed Leakage Power Consumption at $45 \mathrm{~nm}$ at $25^{\circ} \mathrm{C}$

\begin{tabular}{|l|l|l|l|l|}
\hline Gates & \multicolumn{4}{|l|}{ Leakage Power Consumption at 45nm } \\
\hline & 00 & 01 & 10 & 11 \\
\hline NOT & 13.74 & 39.04 & & \\
\hline AND & 55.35 & 84.65 & 64.56 & 90.69 \\
\hline NAND & 19.65 & 42.59 & 23.43 & 76.94 \\
\hline NOR & 27.05 & 46.08 & 38.38 & 59.70 \\
\hline EXOR & 77.06 & 57.62 & 57.62 & 77.06 \\
\hline
\end{tabular}

Table XIV. Proposed Leakage Power Consumption at $45 \mathrm{~nm}$ at $100^{\circ} \mathrm{C}$

\begin{tabular}{|l|l|l|l|l|}
\hline Gates & \multicolumn{5}{|l|}{ Leakage Power Consumption at $45 \mathrm{~nm}$} \\
\hline & 00 & 01 & 10 & 11 \\
\hline NOT & 35.60 & 47.32 & & \\
\hline AND & 103.5 & 170.0 & 148.6 & 129.1 \\
\hline NAND & 26.28 & 64.64 & 44.74 & 93.31 \\
\hline NOR & 69.20 & 55.88 & 46.49 & 63.48 \\
\hline EXOR & 94.28 & 92.74 & 92.74 & 94.28 \\
\hline
\end{tabular}

Table XV. Proposed Leakage Power Consumption at 32nm at $25^{\circ} \mathrm{C}$

\begin{tabular}{|l|l|l|l|l|}
\hline Gates & \multicolumn{4}{|l|}{ Leakage Power Consumption at 32nm } \\
\hline & 00 & 01 & 10 & 11 \\
\hline NOT & 0.598 & 13.24 & & \\
\hline AND & 18.74 & 37.98 & 28.50 & 33.14 \\
\hline NAND & 2.788 & 17.40 & 8.311 & 26.30 \\
\hline NOR & 12.89 & 12.00 & 12.98 & 21.60 \\
\hline EXOR & 25.32 & 23.34 & 23.34 & 25.32 \\
\hline
\end{tabular}

Table XVI. Proposed Leakage Power Consumption at $32 \mathrm{~nm}$ at $100^{\circ} \mathrm{C}$

\begin{tabular}{|l|l|l|l|l|}
\hline Gates & \multicolumn{5}{|l|}{ Leakage Power Consumption (nW) } \\
\hline & 00 & 01 & 10 & 11 \\
\hline NOT & 3.023 & 15.66 & & \\
\hline AND & 103.7 & 170.7 & 159.9 & 112.2 \\
\hline NAND & 23.42 & 66.59 & 56.64 & 56.19 \\
\hline NOR & 15.03 & 20.34 & 27.08 & 26.67 \\
\hline EXOR & 52.13 & 108.4 & 108.4 & 52.13 \\
\hline
\end{tabular}

\section{CONCLUSION}

In nanometer scale CMOS technology, sub-threshold leakage power is compatible to dynamic power consumption, and thus handling leakage power is a great challenge. In this paper, we present a new circuit structure named "stacking with sleepy keeper Approach" to tackle the leakage problem. . It proposes a technique for reducing the leakage current during idle mode of circuit. The proposed technique can be applied on high performance, low power application, where leakage is major concern such as microprocessor, memory units and other portable devices. In future new approach of leakage reduction technique at gate level and block level are expected to give more power saving than the existing approach at CMOS circuit level design.

\section{REFERENCES}

[1] K.Roy and S.C. Prasad, "Low-power CMOS VLSI circuits design". New York: Wiley, 2000, ch.5, pp.214219 .

[2] Y.Taur, T.H. Ning, "Fundamentals of Modern VLSI Devices", Cambridge University Press, New York, 1998.

[3] International Technology Roadmap for Semiconductors (ITRS-

05).http://www.itrs.netLinks/2005ITRS/Design2005.pdf.

[4] Ali Peiravi, Mohammad Asyaei." Robust low leakage controlled keeper by current-comparison domino for wide fan-in gates" INTEGRATION, the VLSI Journal 45 (2012), pp 22-32.

[5] K. Roy, S.Mukhopadhyay, H. Mahmoodi-meimand, "Leakage tolerant mechan- isms and leakage reduction techniques in deep-submicron CMOS circuits", Proceedings of the IEEE 91 (2003), pp. 305-327.

[6] M. Powell, S.-H. Yang, B. Falsafi, K. Roy and T. N. Vijaykumar, "Gated-Vdd: A Circuit Techniqueto Reduce Leakage in Deep submicron Cache Memories," International Symposium on Low Power Electronics and Design, July 2000, pp. 90-95.

[7] Z. Chen, M. Johnson, L. Wei and K. Roy, "Estimation of Standby Leakage Power in CMOS Circuits Considering Accurate Modeling of Transistor Stacks," International Symposium on Low Power Electronics and Design, August 1998, pp. 239-244. 
[8] Kawaguchi, H., Nose, K., and Sakurai, T. “ A Super CutOff CMOS (SCCMOS) Scheme for 0.5-V Supply Voltage with Pico ampere Stand-By Current," IEEE Journal of Solid State Circuits vol.35,n.10, October 2000, pp.1498-1501.

[9] Se Hun Kim, Vincent J. Mooney III, "Sleepy Keeper: a New Approach to Low-leakage Power VLSI Design"

[10] A. Chandrakasan, I. Yang, C. Vieri, and D. Antoniadis, Design Considerations and Tools for Low- Voltage
Digital System Design," In Proceedings of the 33rd Design Automation Conference, pp. 113\{118, 1996\}.

[11] J. Kao, A. Chandrakasan, and D. Antoniadis, ITransistor Sizing Issues and Tools for Multi-threshold CMOS Technology," In Proceedings of the 34th Design Automation Conference, pp. 409\{414, Las Vegas, Nevada, 1997\}. 